\title{
A MONTE CARLO STUDY OF ALTERNATIVE ESTIMATES OF THE COBB-DOUGLAS PRODUCTION FUNCTION:
}

\section{A REJOINDER}

\author{
By J. KMENTA
}

THE MAIN critical comment of Hoch follows from his emphasis on the use of production function estimates for the purpose of testing for efficient allocation of resources. His claim that Klein's estimates are inappropriate in this case is, of course, correct. However, the article was not concerned with any specific field of application-its aim was simply to examine the small sample behavior of several available estimates. Klein's estimates were included because they can legitimately compete with other types of estimates in all applications other than tests for efficiency of allocation. Such applications were thought to be fairly common. Apart from models designed for prediction, economists may be interested in having "good" estimates of marginal productivity of a given type of input for the purpose of comparison with other inputs or other industries or regions. Those concerned with tests for optimality of resource allocation should, of course, limit their attention to estimation methods other than Klein's.

The models used in the experiment were chosen so that, in the opinion of the authors, they would represent reasonably realistic situations. Our choice of the variances of the disturbances was based on the supposition that it is common for the "economic" disturbances to be more, or at best equally, spread out compared with the "technical" disturbance. The same contention was often made in economic literature and was also implied in the selection of combinations considered by Hoch in Table I of his 1958 article. Further, the sum of $a_{1}$ and $a_{2}$ was made equal to 0.9 because it was believed that in a number of industries the returns to scale are fairly close to being constant. Subject to this condition, the value of $a_{1}$ was determined by expediency as mentioned in the text. As for the average firm, it was thought unlikely that it would lie a great distance from the optimum position, which in our models implies a profit of 10 units. Hoch's alternative suggestion of $M \$_{1}=0.50$ and $M \$_{2}=2.00$ would mean a loss of 40 units on the part of the average firm which seems definitely unrealistic in the circumstances. The situation in models $F$ and $G$, in which the average firm is not in the optimum position, was envisaged to be one in which the average firm makes the correct profit-maximizing decisions, hires the appropriate quantity of input 1 , and then finds itself unable to obtain the full 20 units of input 2 . This is different from the interpretation in Hoch's reply since he assumes an immediate adjustment of the quantity of input 1 .

In the final part of his reply Hoch suggests that the "generalized" version 
of his estimation method (i.e., the form which allows for correlation between the "economic" disturbances) would probably be more successful than the "simple" version. With this we agree. Moreover, since the time of carrying out the Monte Carlo study, the writer was able to prove that, for all sample sizes, Hoch's generalized estimates are equivalent to the indirect least squares estimates and, under certain assumptions, are of the maximum likelihood type. The methods differ only in technique of arithmetic computation and render identical results. On the grounds of computational effort the indirect least squares estimates are superior to Hoch's generalized estimates. Because of this equivalence, the generalized method was de facto used in all our models, and Hoch's objection does not apply. The simple estimates of Hoch were examined because they were presented by him for use in empirical work, while the generalized method was not. The results obtained by us should help to throw some light on the properties of the simple estimates if, in fact, they were to be used as proposed in Hoch's paper.

University of Sydney 